\title{
Dedekind sums with predictable signs
}

\author{
by \\ KuRT GiRstMAir (Innsbruck)
}

1. Introduction and main results. Let $b$ and $n$ be integers, $b \neq 0$, with $(b, n)=1$. The (inhomogeneous) Dedekind sum is defined by

$$
s(n, b)=\sum_{k=1}^{|b|}((k / b))((k n / b))
$$

where the symbol $((\ldots))$ has the usual meaning (cf., e.g., [8]). We note the relations

$$
s(n,-b)=s(n, b) \quad \text { and } \quad s(n+b, b)=s(n, b) .
$$

Hence we obtain all Dedekind sums if $b$ is restricted to natural numbers and $n$ to the range $0 \leq n<b$. The general definition, however, will be useful later.

In general, it is not easy to guess what the sign of $s(n, b)$ may be. Rademacher ([7], Satz 3) showed $s(n, b)>0$ for $0<n<\sqrt{b-1}$. In this note we give a considerable generalization of Rademacher's result. Roughly speaking, we shall show that there are a great many intervals $I$ in $[0, b[$ such that $s(n, b)$ takes a predictable and fixed sign for each $n \in I$.

To this end we fix the natural number $b$ for the time being. Let $d<b$ be another natural number. Define

$$
\alpha_{d}=-\frac{b(d-1)(d-2)}{2(b d-1)}, \quad \beta_{d}=\frac{(b-d)^{2}}{b d-1}
$$

and

$$
\gamma_{d}=\alpha_{d}+\sqrt{\beta_{d}+\alpha_{d}^{2}}
$$

the square root being positive. To each fraction $c / d, c \in \mathbb{Z},(c, d)=1$, we attach an interval of length $2 \gamma_{d} / d$ with midpoint $b \cdot c / d$, namely,

$$
I(c, d)=\left\{x \in \mathbb{R}:|x-b \cdot c / d|<\gamma_{d} / d\right\} .
$$

1991 Mathematics Subject Classification: Primary 11F20. 
Both "half-intervals"

$I(c, d)^{-}=\{x \in I(c, d): x<b \cdot c / d\}, \quad I(c, d)^{+}=\{x \in I(c, d): x>b \cdot c / d\}$ are nonempty. Moreover, each number $n \in \mathbb{Z},(n, b)=1$, lying in $I(c, d)$ belongs to one of these half-intervals. Otherwise $n=b \cdot c / d$, but then $d \mid b$ because of $(c, d)=1$, so $b / d$ divides $(n, b)$, which is 1 . This is impossible since $b / d>1$. Our first main result is

THEOREM 1. As above, let $d<b$ be natural numbers and $c$ an integer with $(c, d)=1$. Let $n$ be an integer in $I(c, d),(n, b)=1$. Then $s(n, b)<0$ if $n \in I(c, d)^{-}$, and $s(n, b)>0$ if $n \in I(c, d)^{+}$.

If $d=1$, then $\alpha_{d}=0$ and $\beta_{d}=b-1$, so $\gamma_{d} / d=\gamma_{d}=\sqrt{b-1}$ and $\left.I(0,1)^{+}=\right] 0, \sqrt{b-1}[$. Therefore, the case $d=1$ of Theorem 1 contains Rademacher's above-mentioned result. In view of (1) and the well-known identity

$$
s(-n, b)=-s(n, b),
$$

it is clear that Rademacher's theorem is equivalent to this special case of Theorem 1.

We look at the intervals $I(c, d)$ more closely. It suffices, of course, to consider only those parts of them that are contained in $[0, b[$. Apart from the half-intervals $I(0,1)^{+}$and $\left.I(1,1)^{-}=\right] b-\sqrt{b-1}, b[$, these parts are just the complete intervals $I(c, d), 2 \leq d<b$, with $1 \leq c<d,(c, d)=1$. It will be shown below that, if $b \geq 4$, then

$$
\sqrt{b / d^{3}}-1<\gamma_{d} / d<\sqrt{b / d^{3}}
$$

(cf. Lemma 2, (20), (21)). This means that the length $2 \gamma_{d} / d$ of an interval $I(c, d)$ is of order of magnitude $\approx \sqrt{b}$ if $d^{3}$ is small relative to $b$. In this case we say that $I(c, d)$ is "large". Obviously, large intervals contain many integers $n$. There is no reason, however, to rule out "small" intervals. It follows from (5) that $I(c, d),(c, d)=1$, contains at least one integer if $d<(3 / 4) b^{1 / 3}$; and it turns out that at least some of the intervals $I(c, d)$ contain an integer as long as $d<\sqrt{b}$. Conversely, $I(c, d) \cap \mathbb{Z}$ is empty for $d \geq \sqrt{b}$ (see the remark following the proof of Theorem 1 ). In view of this, it is natural to study the subset

$$
R(b)=I(0,1)^{+} \cup I(1,1)^{-} \cup \bigcup_{2 \leq d<\sqrt{b}} \bigcup_{\substack{1 \leq c<d \\(c, d)=1}} I(c, d)
$$

of $[0, b[$. The set $R(b)$ will be called the region of predictable sign. It would be desirable to know the number

$$
S(b)=|R(b) \cap \mathbb{Z}|
$$

of integers in $R(b)$. We show 
THEOREM 2. If $b$ is large enough, then

$$
1.8 \cdot b^{2 / 3}<S(b)<4.75 \cdot b^{2 / 3} .
$$

According to Theorem 2 the number of integers in the region of predictable sign is substantially larger than the size of large intervals $I(c, d)$. Both constants in Theorem 2 are rather pessimistic - the true order of magnitude of $S(b)$ seems to be $\approx 3.1 \cdot b^{2 / 3}$. Further details on the growth of $S(b)$ can be found in Sections 3 and 4 .

The diagrams below may give an idea of the behaviour of the values of $s(n, b)$ inside and outside $R(b)$. They display the case $b=1009$, a prime, where $S(b)=266$. The small circles represent pairs $(n, 12 s(n, b))$. Observe that

$$
|12 s(n, b)|<b
$$

holds for arbitrary numbers $n, b$ with $(n, b)=1$ (cf. (14)). In the first diagram the values $n=1$ and $n=b-1$ have been omitted-just to save space, since these are the only ones with $|12 s(n, b)|$ close to $b$; for any other $n,|12 s(n, b)|<b / 2$. The diagrams suggest that $R(b)$ contains all integers $n$ for which $|s(n, b)|$ is "large" but not only these; conversely, the complement $[0, b[\backslash R(b)$ seems to consist only of numbers $n$ with $|s(n, b)|$ small. Indeed, our computations show that $|12 s(n, b)|$ seldom exceeds $\sqrt{b}$ if $n$ is not in $R(b)$, whereas there are many numbers $n$ in $R(b)$ with $|12 s(n, b)|<\sqrt{b}$.

2. The proof of Theorem 1. Theorem 1 is based on a relation for Dedekind sums (Lemma 1) that generalizes the usual three-term relation of Rademacher [6]. This lemma is a consequence of the transformation law of the logarithm of Dedekind's $\eta$-function. Relations of this more general type were given by Dieter [4] and frequently used by Bruggeman (cf., e.g., [3], formula (3.1); [2], part 2.3). Nevertheless it seems that these relations are not commonly known (cf. the rediscovery in [5]). For the convenience of the reader we include a short proof, since it may be toilsome to adapt the results of [4] to the situation considered here.

Let $d, b$ be natural numbers and $n, c$ integers with $(n, b)=(c, d)=1$. We write

$$
n-b \cdot c / d=q / d,
$$

where $q$ is an integer. Suppose $q \neq 0$ and put

$$
\varepsilon=\operatorname{sign}(q)(\in\{ \pm 1\}) .
$$

Moreover, let $j$ and $k$ be integers such that

$$
-c j+d k=1
$$

and put

$$
r=-b k+n j
$$




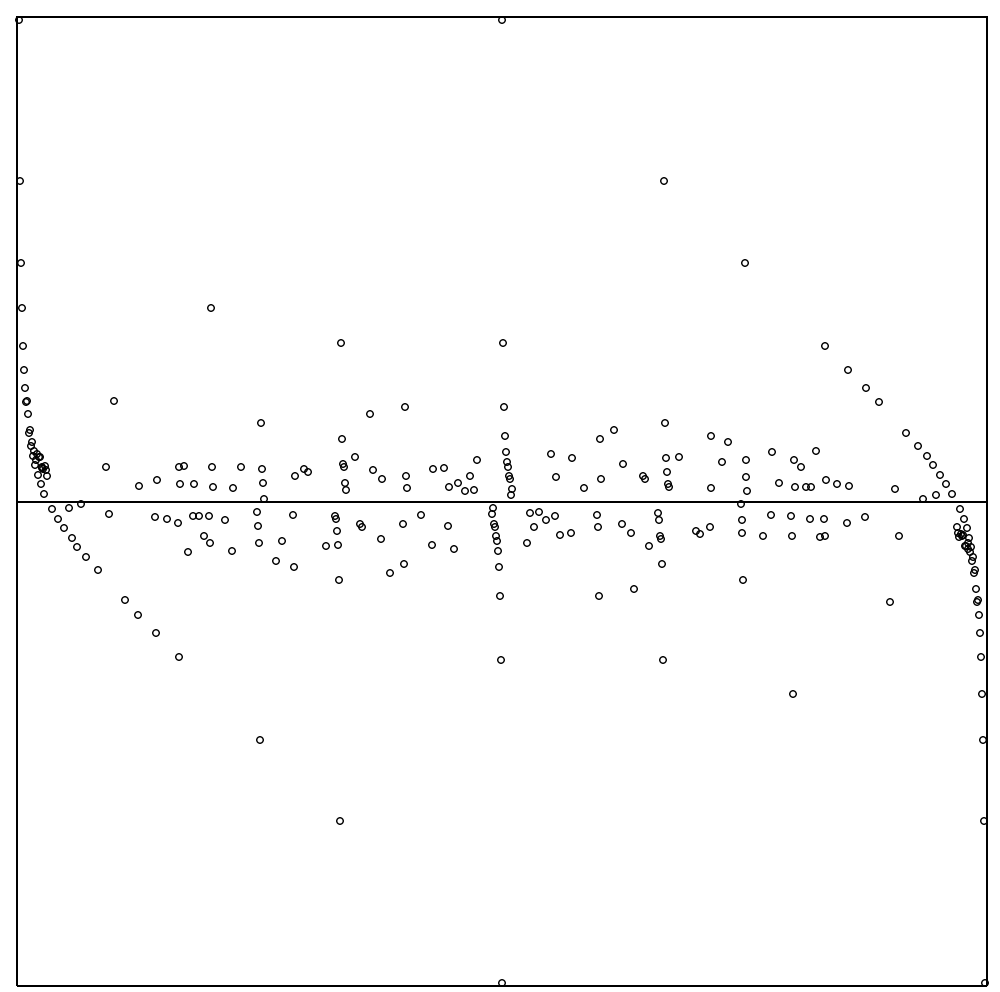

$b=1009:$ the values of $12 s(n, b)$ for $n \in R(b), n \neq 1, b-1$

LEMMA 1. In the above situation,

$$
12 s(n, b)=12 s(c, d)+\varepsilon \cdot 12 s(r, q)+\frac{b^{2}+d^{2}+q^{2}}{b d q}-3 \varepsilon .
$$

Proof. The transformation law of the logarithm of Dedekind's $\eta$-function says

$$
\Phi(A B)=\Phi(A)+\Phi(B)-3 \operatorname{sign}(A) \operatorname{sign}(B) \operatorname{sign}(A B)
$$

(cf. [8], pp. $49 \mathrm{ff}$ ). Here $A, B$ denote matrices in $\mathrm{SL}(2, \mathbb{Z})$ and $\Phi$ and sign are defined in the following way: If

$$
A=\left(\begin{array}{ll}
\alpha & \beta \\
\gamma & \delta
\end{array}\right)
$$

then $\operatorname{sign}(A)=\operatorname{sign}(\gamma)(\in\{0, \pm 1\})$, and

$$
\Phi(A)= \begin{cases}\beta / \delta & \text { if } \gamma=0 \\ (\alpha+\delta) / \gamma-12 \operatorname{sign}(A) s(\delta, \gamma) & \text { otherwise }\end{cases}
$$


In our situation we put

$$
A=\left(\begin{array}{ll}
u & v \\
b & n
\end{array}\right), \quad B=\left(\begin{array}{cc}
-c & -k \\
d & j
\end{array}\right),
$$

where $u, v$ are integers such that

$$
u n-v b=1 .
$$

On applying (10) one readily obtains (observe (4))

$$
\begin{aligned}
12 s(n, b)= & 12 s(-j, d)+\varepsilon \cdot 12 s(r, q) \\
& +(u c-v d+b k-j n) / q+(u+n) / b+(j-c) / d-3 \varepsilon .
\end{aligned}
$$

Because of $(9),-c j \equiv 1 \bmod d$, and a well-known identity (cf. [8], p. 26) says

$$
s(-j, d)=s(c, d) .
$$

Therefore, the right side of (12) has the desired shape if only the sum of the three fractions equals $\left(b^{2}+d^{2}+q^{2}\right) /(b d q)$. But this follows from a short calculation which takes the identities (9) and (11) into account.

Proof of Theorem 1. We consider the case $n>b \cdot c / d$ first. Let $q$ be defined by (8), so $q>0$ and $\varepsilon=1$. By the lemma, $s(n, b)>0$ holds if, and only if,

$$
12 s(c, d)+12 s(r, q)+\left(b^{2}+d^{2}+q^{2}\right) /(b d q)-3>0 .
$$

Next we apply the estimate

$$
|12 s(x, y)| \leq(|y|-1)(|y|-2) /|y|,
$$

which holds for arbitrary coprime integers $x, y, y \neq 0$ (cf. [7]). Thereby, the left side of (13) is $>0$ if only

$$
-(d-1)(d-2) / d-(q-1)(q-2) / q+\left(b^{2}+d^{2}+q^{2}\right) /(b d q)-3>0 .
$$

This is the same as saying that $f(d, q)<0$, where $f(d, q)$ is the polynomial defined by

$$
f(d, q)=b q(d-1)(d-2)+b d(q-1)(q-2)-\left(b^{2}+d^{2}+q^{2}\right)+3 b d q .
$$

We consider $f(d, q)$ as a polynomial in $q$ only and note

$$
f(d, q) /(b d-1)=q^{2}-2 \alpha_{d} \cdot q-\beta_{d}
$$

(cf. (2)). Hence $f(d, q)$ is negative if, and only if, $q$ lies between the zeros

$$
\alpha_{d} \pm \sqrt{\beta_{d}+\alpha_{d}^{2}}
$$

of $f(d, q)$. Since $q$ is positive, this means nothing but $q<\gamma_{d}$ (cf. (3)) and $n \in I(c, d)^{+}$. 
In the case $n<b \cdot c / d$ we have $q<0$ and $\varepsilon=-1$. One shows, in the same way, that $s(n, b)<0$ if $f(d,|q|)<0$, which means $|q|<\gamma_{d}$ and $n \in I(c, d)^{-}$.

REMARK. We draw the reader's attention to the fact that the definition (15) of $f(d, q)$ is symmetric in $d$ and $q$. This allows rephrasing the assertion " $n \in I(c, d)$ " in another way. Indeed, let $q$ be defined by (8). Then " $n \in$ $I(c, d)$ " is the same as saying $|q|<\gamma_{d}$ or $f(d,|q|)<0$. This, however, is equivalent to $f(|q|, d)<0$ or $d<\gamma_{|q|}$. Now the (still unproved) estimate (5), applied to $\gamma_{|q|}$, gives $\gamma_{|q|}<\sqrt{b /|q|}$; so $n \in I(c, d)$ can hold only if

$$
d<\sqrt{b /|q|}
$$

In particular, $I(c, d) \cap \mathbb{Z}$ is empty if $d \geq \sqrt{b}$ - as we said in Section 1 .

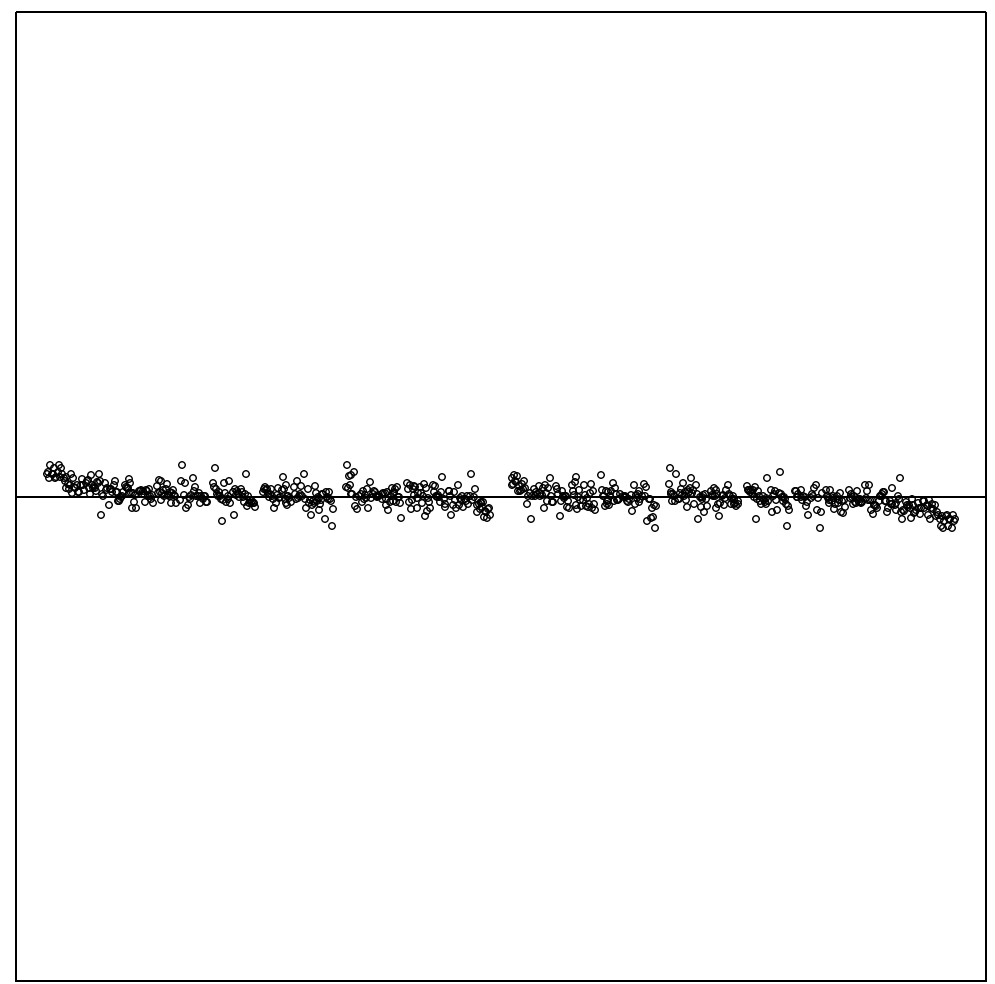

$b=1009$ : the values of $12 s(n, b)$ for $n \notin R(b), 1 \leq n<b$

3. The sums $S_{1}(b)$ and $S_{2}(b)$. Obviously, the sets $I(c, d) \cap \mathbb{Z}$ must be pairwise disjoint (otherwise we get a contradiction to Theorem 1). Hence 
the definitions (6) and (7) show

$$
S(b)=\sum_{1 \leq d<\sqrt{b}} S_{b, d}
$$

with

$$
S_{b, d}=\sum_{\substack{0 \leq c<d \\(c, d)=1}}|I(c, d) \cap \mathbb{Z}| .
$$

The proof of Theorem 2 is based on the separate treatment of the sums

$$
S_{1}(b)=\sum_{1 \leq d<b^{1 / 3}} S_{b, d}, \quad S_{2}(b)=\sum_{b^{1 / 3} \leq d<\sqrt{b}} S_{b, d} .
$$

In fact, some of the estimates used for the first sum do not work in the case of the second and conversely. We shall show

Proposition 1. (a) For each sufficiently large natural number $b$,

$$
\frac{18}{\pi^{2}} b^{2 / 3}-\frac{9}{8} \sqrt{b}<S_{1}(b)<\frac{27}{\pi^{2}} b^{2 / 3} .
$$

(b) If $b$ is large enough, then

$$
S_{2}(b)<2 \cdot b^{2 / 3}+b^{0.51} .
$$

Both parts (a) and (b) together yield

$1.8 \cdot b^{2 / 3}<S_{1}(b) \leq S(b)=S_{1}(b)+S_{2}(b)<2.74 \cdot b^{2 / 3}+2.01 \cdot b^{2 / 3}=4.75 \cdot b^{2 / 3}$ and hence Theorem 2. Next we list some estimates needed for the proof of Proposition 1.

LeMma 2. Let $d<b$ be natural numbers and $\alpha_{d}, \beta_{d}, \gamma_{d}$ as in (2) and (3). Then

$$
\begin{gathered}
(d-3) / 2<\left|\alpha_{d}\right|<d / 2, \\
\sqrt{b / d}-\sqrt{d / b}<\sqrt{\beta_{d}}<\sqrt{b / d}, \\
\gamma_{d} / d>\sqrt{b / d^{3}}-1 / \sqrt{b d}-1 / 2, \\
\gamma_{d} / d<\sqrt{b / d^{3}} .
\end{gathered}
$$

Finally, if $12 \leq d<b$,

$$
\gamma_{d}<\left(1+\frac{4}{d}\right) \frac{b}{d^{2}}
$$

Pro of. Observe that

$$
2\left|\alpha_{d}\right|=\frac{b(d-1)(d-2)}{b d-1} \geq \frac{b(d-1)(d-2)}{b d}>\frac{d^{2}-3 d}{d} .
$$


Moreover, if $d=1,\left|\alpha_{d}\right|<d / 2$ is true. For $d \geq 2$,

$$
2\left|\alpha_{d}\right|=\frac{(d-1)(d-2)}{d-1 / b} \leq d-2<d
$$

This proves (18). In order to prove (19), note

$$
\beta_{d}=\frac{(b-d)^{2}}{b d-1}>\frac{(b-d)^{2}}{b d},
$$

hence $\sqrt{\beta_{d}}>(b-d) / \sqrt{b d}=\sqrt{b / d}-\sqrt{d / b}$. Further,

$$
\beta_{d}=\frac{b-d}{d} \cdot \frac{b-d}{b-1 / d} \leq \frac{b-d}{d}<b / d .
$$

Assertion (20) is immediate from the definition (3) of $\gamma_{d}$, the upper bound for $\left|\alpha_{d}\right|$ and the lower bound for $\sqrt{\beta_{d}}$ which are displayed in (18), (19), respectively. In order to show (21) we use

$$
\sqrt{\beta_{d}+\alpha_{d}^{2}} \leq \sqrt{\beta_{d}}+\left|\alpha_{d}\right|
$$

which can be verified by squaring. This gives $\gamma_{d} \leq \sqrt{\beta_{d}}<\sqrt{b / d}$, by (19). Finally, (19) implies

$$
\gamma_{d}<-\left|\alpha_{d}\right|+\sqrt{b / d+\alpha_{d}^{2}}
$$

We show

$$
\sqrt{b / d+\alpha_{d}^{2}} \leq(1+4 / d) \cdot b / d^{2}+\left|\alpha_{d}\right|,
$$

which yields (22). However,

$$
\left(\left(1+\frac{4}{d}\right) \frac{b}{d^{2}}+\left|\alpha_{d}\right|\right)^{2} \geq \alpha_{d}^{2}+2\left|\alpha_{d}\right|\left(1+\frac{4}{d}\right) \frac{b}{d^{2}} \geq \alpha_{d}^{2}+(d-3)\left(1+\frac{4}{d}\right) \frac{b}{d^{2}},
$$

by (19). One checks that $(d-3)(1+4 / d) \geq d$ whenever $d \geq 12$ and obtains (23).

Proof of Proposition 1(a). Since $I(c, d)$ is an open interval of length $2 \gamma_{d} / d$, it is clear that

$$
2 \gamma_{d} / d-1 \leq|I(c, d) \cap \mathbb{Z}| \leq 2 \gamma_{d} / d+1
$$

Therefore, (16) gives

$$
\varphi(d)\left(2 \gamma_{d} / d-1\right) \leq S_{b, d} \leq \varphi(d)\left(2 \gamma_{d} / d+1\right),
$$

where $\varphi(\ldots)$ denotes Euler's function. Now the assertion follows from (17), (20), (21), and the following three formulas that hold for large $b$ :

$$
\sum_{1 \leq d<b^{1 / 3}} \frac{\varphi(d)}{d^{3 / 2}}=\frac{12}{\pi^{2}} b^{1 / 6}+C+O\left(b^{-1 / 6} \log b\right),
$$


with $-0.56<C<0$,

$$
\sum_{1 \leq d<b^{1 / 3}} \frac{\varphi(d)}{\sqrt{d}}=O(\sqrt{b}),
$$

and

$$
\sum_{1 \leq d<b^{1 / 3}} \varphi(d)=\frac{3}{\pi^{2}} b^{2 / 3}+O\left(b^{1 / 3} \log b\right) .
$$

Of these, (25) is quite elementary since its left side is

$$
\leq \sum_{1 \leq d<b^{1 / 3}} \sqrt{d}=O\left(\left(b^{1 / 3}\right)^{3 / 2}\right)
$$

The remaining two formulas are applications of standard results (cf. [1], p. 62, Theorem 3.7, and p. 71, Exercise 7).

The upper bound for $S_{b, d}$ given in (24) is not good enough for the proof of Proposition 1(b). Instead, we shall use

Lemma 3 . Let $1 \leq d<b$. Then

$$
S_{b, d} \leq 2 \gamma_{d}+(d, b) \text {. }
$$

Proof. By (24), the assertion is true for $d=1$. Suppose, henceforth, $d \geq 2$. $S_{b, d}$ is the number of all integers $n, 0 \leq n<b$, such that $n \in I(c, d)$ holds for some $c, 0 \leq c<d,(c, d)=1$. But saying " $n \in I(c, d)$ " is the same as saying

$$
|n d-b c|<\gamma_{d}
$$

For every $k \in \mathbb{Z}$ define $(k)_{b} \in \mathbb{Z}$ by the conditions

$$
(k)_{b} \equiv k \bmod b, \quad-b / 2 \leq(k)_{b}<b / 2
$$

(so $(k)_{b}$ is a certain representative of the congruence class of $k \bmod b$ ). Now (21), together with the inequalities $1<\sqrt{b / d}<b / d \leq b / 2$, yields $\gamma_{d}<b / 2$. Consequently, (26) can hold only if $\left|(n d)_{b}\right|<\gamma_{d}$. But then

$$
S_{b, d} \leq\left|\left\{n: 0 \leq n<b,\left|(n d)_{b}\right|<\gamma_{d}\right\}\right| .
$$

We write $\delta=(d, b), d=d^{\prime} \delta$, and $b=b^{\prime} \delta$. The identity

$$
(n d)_{b}=\left(n d^{\prime}\right)_{b^{\prime}} \cdot \delta
$$

readily shows

$$
\begin{aligned}
\mid\left\{n: 0 \leq n<b,\left|(n d)_{b}\right|\right. & \left.<\gamma_{d}\right\} \mid \\
& =\delta \cdot\left|\left\{n: 0 \leq n<b^{\prime},\left|\left(n d^{\prime}\right)_{b^{\prime}}\right|<\gamma_{d} / \delta\right\}\right| .
\end{aligned}
$$

Since $\left(d^{\prime}, b^{\prime}\right)=1$, the map $n \mapsto\left(n d^{\prime}\right)_{b^{\prime}}$ is injective on $\left\{n: 0 \leq n<b^{\prime}\right\}$. Thus,

$$
\left|\left\{n: 0 \leq n<b^{\prime},\left|\left(n d^{\prime}\right)_{b^{\prime}}\right|<\gamma_{d} / \delta\right\}\right| \leq 2 \gamma_{d} / \delta+1 .
$$

By (27) and (28), $S_{b, d} \leq 2 \gamma_{d}+\delta$, as desired. 
Proof of Proposition 1(b). Lemma 3 yields

$$
S_{2}(b) \leq 2 \sum_{b^{1 / 3} \leq d<\sqrt{b}} \gamma_{d}+\sum_{b^{1 / 3} \leq d<\sqrt{b}}(d, b) .
$$

The second sum is dominated by

$$
\sum_{1 \leq d<\sqrt{b}}(d, b) \leq \sum_{d \mid b} d \cdot|\{n: 1 \leq n<\sqrt{b}, d \mid n\}| \leq \sum_{d \mid b} d \cdot \sqrt{b} / d=\sqrt{b} \sum_{d \mid b} 1,
$$

which is $\leq b^{0.51}$ as soon as $b$ is large enough. As to the first sum, we assume $b^{1 / 3} \geq 12$ and use (22) together with the formulas

$$
\sum_{d \geq b^{1 / 3}} 1 / d^{2}=b^{-1 / 3}+O\left(b^{-2 / 3}\right), \quad \sum_{d \geq b^{1 / 3}} 1 / d^{3}=O\left(b^{-2 / 3}\right)
$$

(cf. [1], pp. $55 \mathrm{ff}$ ). This concludes the proof.

4. Additional observations. The proof of Theorem 2 might suggest that the sum $S_{2}(b)$ does not actually play a role for the growth of $S(b)$-for instance, we did not even use $S_{2}(b)>0$. Numerical examples, however, indicate that $S_{1}(b)$ and $S_{2}(b)$ have the same order of magnitude, namely $b^{2 / 3}$ (cf. Table 1, which displays some cases in which $b$ is a prime).

If one assumes that the behaviour of the region $R(b)$ relative to integers is "random", one expects that its (usual) measure $\varrho(b)$ is close to $S(b)$. This is the case in the examples listed below. Here we note that the intervals $I(c, d)$ are mutually disjoint-so this is true not only for the integers in these intervals. Therefore,

$$
\varrho(b)=\sum_{1 \leq d<\sqrt{b}} \varphi(d) \cdot 2 \gamma_{d} / d .
$$

However, we abstain from proving the said disjointness, some details being fairly toilsome. With the aid of Lemma 2 it is not difficult to show

$$
\varrho(b)<\frac{36}{\pi^{2}} b^{2 / 3} \text {. }
$$

The right side seems to be a more realistic upper bound for $S(b)$ than that of Theorem 2.

Table 1

\begin{tabular}{crrrrr}
\hline$b$ & \multicolumn{1}{c}{$S(b)$} & \multicolumn{1}{c}{$S_{1}(b)$} & \multicolumn{1}{c}{$S_{2}(b)$} & \multicolumn{1}{c}{$\varrho(b)$} & $\left(36 / \pi^{2}\right) \cdot b^{2 / 3}$ \\
\hline $10^{5}+3$ & 6338 & 4378 & 1960 & 6308.8 & 7858.6 \\
$10^{6}+3$ & 30210 & 20716 & 9494 & 30123.4 & 36475.7 \\
$10^{7}+19$ & 143010 & 97536 & 45474 & 142693.3 & 169305.1 \\
$10^{8}+7$ & 672954 & 457150 & 215804 & 671954.9 & 785843.6 \\
$10^{9}+7$ & 3153674 & 2136180 & 1017494 & 3150637.2 & 3647562.6 \\
\hline
\end{tabular}


One may ask whether the intervals $I(c, d)$ of Theorem 1 are "largest possible" or, conversely, whether they can be extended to larger intervals with the same behaviour of the sign. Indeed, an extension is possible in individual cases but not in general. Rademacher ([7], Satz 1) showed that $s(n, b)=0$ if $n=\sqrt{b-1}$, so the intervals $I(c, 1)$ cannot be enlarged if $b-1$ happens to be a square. In addition, we investigated many numbers $d>1$ and found numerous examples of sign changes of $s(n, b)$ as soon as $n$ passes one of the boundaries of $I(c, d)$.

The behaviour of $s(n, b)$ inside the intervals $I(c, d)$ is explained, partially at least, by the following observation, which is based on Lemma 1, too (cf. also [3], part 2.4): If $d$ is small relative to $b$ and $n$ is close to the midpoint $b \cdot c / d$ of $I(c, d)$, the point $(n, 12 s(n, b))$ is close to the point $(x, y)$ of the hyperbola

$$
(x-b \cdot c / d) \cdot y=b / d^{2}
$$

with $x=n$. In particular, the sign of $s(n, b)$ agrees with that of $y$. When $n$ moves away from $b \cdot c / d$, the point $(n, 12 s(n, b))$ may gradually leave its companion $(n, y)$-however, it must not cross the asymptote $y=0$ of the hyperbola as long as $n$ remains inside $I(c, d)$. The reader may inspect the cases $d=2,3$ in the first diagram above.

Finally, we observe that the estimate (21) implies

$$
|n / b-c / d|<1 /\left(2 d^{2}\right)
$$

for any $n \in I(c, d)$ if only $b \geq 12$ (recall that $d$ must be $<\sqrt{b}$ if $I(c, d)$ is nonempty). Therefore, the fraction $c / d$ is a convergent of $n / b$ according to Legendre's criterion. In order to test whether a given number $n,(n, b)=1$, is in the region $R(b)$ one may proceed as follows: Compute the continued fraction of $n / b$ and check whether some convergent $c / d, d<\sqrt{b}$, satisfies (26). If this is the case, $n$ is in $I(c, d)$ and hence in $R(b)$, otherwise $n$ lies outside $R(b)$.

\section{References}

[1] T. M. A postol, Introduction to Analytic Number Theory, Springer, New York, 1976.

[2] R. Bruggeman, On the distribution of Dedekind sums, in: Contemp. Math. 166, Amer. Math. Soc., 1994, 197-210.

[3] -, Dedekind sums for Hecke groups, Acta Arith. 71 (1995), 11-46.

[4] U. Dieter, Beziehungen zwischen Dedekindschen Summen, Abh. Math. Sem. Univ. Hamburg 21 (1957), 109-125.

[5] J. E. Pommersheim, Toric varieties, lattice points, and Dedekind sums, Math. Ann. 295 (1993), 1-24.

[6] H. Radem acher, Generalization of the reciprocity formula for Dedekind sums, Duke Math. J. 21 (1954), 391-397.

[7] _, Zur Theorie der Dedekindschen Summen, Math. Z. 63 (1956), 445-463. 
[8] H. Rademacher and E. Grosswald, Dedekind Sums, Carus Math. Monographs 16, Math. Assoc. Amer., 1972.

Institut für Mathematik

Universität Innsbruck

Technikerstr. 25/7

A-6020 Innsbruck, Austria

E-mail: Kurt.Girstmair@uibk.ac.at

Received on 18.7 .1997

and in revised form on 16.9.1997 